\title{
An Advanced Qos Analysis ANd Evaluation METHOD FOR MOBILE INTERNET ACCESS
}

\author{
F. Beritelli, C. Rametta, A. Raspanti*, M. Russo ${ }^{\circ}$, F. Scaglione ${ }^{\circ}$ and G. Spallina* \\ Dipartimento di Ingegneria Elettrica, Elettronica e Informatica,University of Catania, \\ V.le A. Doria, 6 - 95125 Catania, Italy \\ * HELILAB S.R.L. Via IV Novembre, 130 F - 95022 Aci Catena (CT) - Italy \\ ${ }^{\circ}$ VICOSYSTEMS S.R.L. V. le O. Da Pordenone, 33 -95128 Catania - Italy
}

\begin{abstract}
The paper proposes a new method for the analysis and evaluation of the Quality of Service (QoS) in a mobile Internet access scenario. In particular, the paper proposes a throughput evaluation method based on PathChirp algorithm. The end-to-end bandwidth was estimated by means of the Self Loading of Periodic Streams (SloPS) technique. The obtained measurements were then analyzed by estimating the degree of correlation with other parameters that characterize the data transmission such as power, round trip time, etc. Finally, in order to have greater spatial resolution performance guaranteed by an Internet service provider, a 3D reconstruction method based on using drones is proposed and some preliminary results are discussed.
\end{abstract}

\section{KEYWORDS}

Radio Access Evaluation; 3G-4G Networks, Throughput Evaluation Algorithms; RAN KPIs Correlation.

\section{INTRODUCTION}

Mobile Internet is growing rapidly due to low setup costs and decrease in price. We live in a wireless world, where the demand for the Internet on our laptops, tablets and smart phones is a necessity anywhere we go. However, not everyone has access to the data speed they require. The main reason for slow Internet speed is weak signal strength due to inadequate radio coverage in critical areas such as those in the suburbs.

The paper proposes a new method for the analysis and evaluation of the Quality of Service (QoS) in a mobile Internet access scenario. In particular, the paper proposes a throughput evaluation method based on PathChirp algorithm [1]. The end-to-end bandwidth was estimated by means of the Self Loading of Periodic Streams (SLoPS) technique [2][3].

Part of the work done in this paper was focused on the identification of an alternative technique to simple FTP, but still able to provide an accurate estimate of the throughput. This parameter is often measured through an FTP session, by sending a file and analyzing the time it takes to transfer it, calculating thus the file transfer speed, expressed in bit/s. The selection of too small size files could speed the measurement time, but it could lead to an inaccurate estimate of throughput, conversely the selection of large files would provide a more accurate estimate of throughput, but it would require more time to complete the transfer and, obtaining the result, still rank higher in low bandwidth availability. 
To this end, we have analyzed the PathLoad [2] and PathChirp techniques, both techniques based on a client-server paradigm, and able to provide an estimate of the available bandwidth end-toend.

By comparing these techniques with the FTP technique, it was found that the data rate of the measured values with PathChirp are very close to the measured values with the FTP technique, but with the advantage of significantly faster convergence time.

Once the technique to derive the throughput estimates was identified, measurements on certain KPIs (Key Performance Indicators) were taken and presented in Section III. Following these measurements, the correlation values were also calculated between the received signal strength and other parameters measured, in order to assess how the latter were influenced by the signal strength level received.

In order to estimate the QoS in a Mobile Internet context, nowadays many measurements are carried out in motion on board a motor vehicle by identifying various test points along the route in which the measurements are taken. Obviously there are limitations: the measurements are limited only to areas accessible by a motor vehicle and they provide estimates of the QoS offered to a preset altitude from ground level, thus not providing any suggestions as to the possible variations in height.

The final idea is to apply this measuring mechanism aboard a drone, with the considerable advantage of being able to take measurements in areas not easily accessible and above all provide an overview of QoS in a 3D scene, with drone being able to take measurements even in height.

\section{STATE OF ART}

On the one hand, recently we have been assisting to a relevant increase in the drone use in many different scenarios: inspection of large infrastructure for remote damage assessment and monitoring purposes [4], support for emergency operations, such as finding missing persons [5], support in rapid and effective organization of disaster recovery operations [6][7][8], applications for smart city contexts [9][10], and many other scenarios.

On the other hand, mobile networks are gradually becoming the predominant source of Internet access, so developers, researchers, network operators, and regulatory communities have taken an increased interest in measuring end-to-end mobile network performance [11][12].

The first approach to the use of drones in mobile network performance evaluation was already taken by employing drones carrying smartphones with network testing applications to analyze the KPIs and estimate the offered QoS [13].

In this paper, however, we propose a new method of network mobile performance evaluation, which is not based on a simple smartphone, but rather on a dedicated and optimized prototype installed on a drone.

\section{DRONE-INTERNET TECHNOLOGY}

Drones for civilian applications (less than $10 \mathrm{~kg}$ in weight) have been one of the topics of great interest in recent years (Fig. 1). The application contexts are numerous and applications range from video footage, 3D reconstructions of buildings and architectural structures, to playful contexts, 
etc.As is well known, while our increasingly hyperconnected world brings many benefits, it also exacerbates inequalities for the share of the global population that has limited or no Internet access.

In some scenarios it is also essential to ensure Internet access as a backup to traditional solutions, consider, for special events such as earthquakes, the ground war actions, etc. where it is vital to ensure immediate coverage of Internet access.

Another equally important application area covers the drone as a multi-sensor mobile system that allows measurement of a parameter set. The benefit is a completely innovative spatial sampling method that becomes fundamental in many applications in which a greater spatial resolution of the measured data may be necessary.

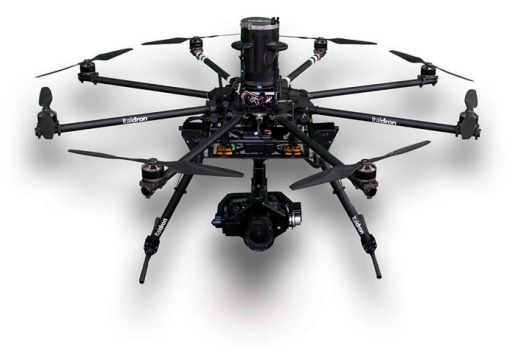

Figure 1. Drone for civilian applications

Recently, much has been said of the drone-Internet combination as an innovative technology to expand Internet access in areas not reached by the traditional radio coverage for the delivery of services via Internet. Think of Google's top-secret Project SkyBender testing the viability of millimeter wave technology beamed from solar-powered Titan drones, in order to deliver 5G internet and other internet-over-the-air projects.

Mountain View has reportedly been experimenting with millimeter wave-based Internet. Millimeter waves are believed to be capable of transmitting data 40 times faster than LTE and could become the technology behind $5 \mathrm{G}$ internet.

Lately, though, two initiatives - Google Project Loon and Mark Zuckerberg's internet.org have drawn widespread attention to the challenge of using alternative delivery platforms to bring low cost broadband access to unserved or underserved parts of the world.

Project Loon plans to bring Internet access to remote locations via the network of high-altitude balloons. As Google describes it, "People can connect to the balloon network using a special Internet antenna attached to their building."

Internet.org offers a similar approach, except instead of balloons, it envisions using drones as the delivery platform.

The benefits of all-wireless network in the sky are clear. Such a network would be far less expensive, far less disruptive and it would take far less time to build than implementing a wired / land based infrastructure over very large swaths of the earth where no communications infrastructure currently exists. 


\section{Description Of The Prototype}

To perform our tests we developed a prototype by using a small form factor system boards optimized for wireless routing and network applications, an ALIX2D2 board [14], equipped with a $500 \mathrm{MHz}$ AMD Geode LX800 CPU and 256 MB DDR DRAM on board; a USB Internet key has been used to provide cellular connectivity [15][16]; ZeroShell[17] has been used as the operative system whereas proprietary scripts have been developed by using bash and python language [18] to evaluate the available end-to-end bandwidth between the prototype and a remote server acting as the end point of the communication, RSSI, Round Trip Time (RTT) and standard deviation of RTT. A picture of the hardware employed to realize our prototype is shown in Figure 2.

Two algorithms have been implemented on board the prototype: PathLoad and PathChirp. Both algorithms evaluate the end-to-end bandwidth by means of the Self Loading of Periodic Streams (SloPS) technique and they are based on a client-server paradigm: the sender process runs on the mobile equipment whereas the receiver process runs on a remote host.

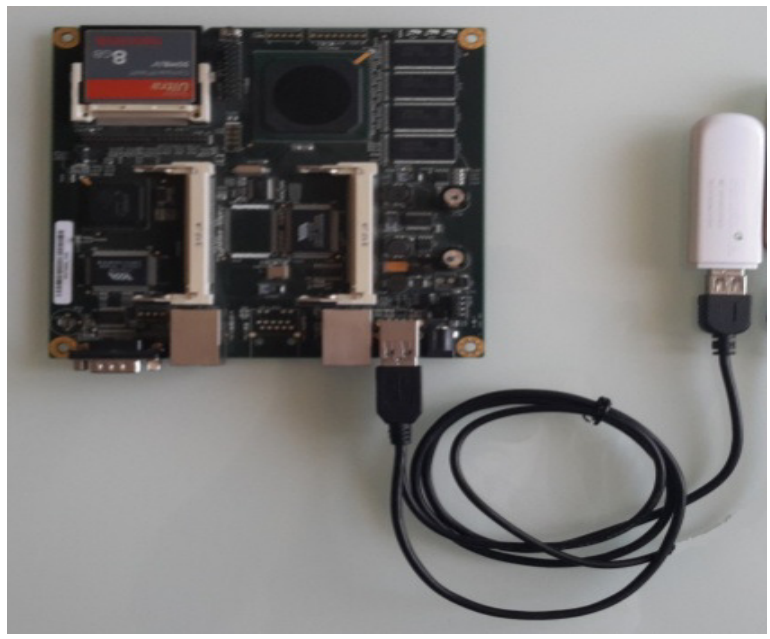

Figure 2. The prototype employed for our test bed.

The working principle of the Self Loading of Periodic Streams is very simple: it is based on the periodic transmission of packet streams from the source to the destination and on the consequent measurement of the One Way Delay (OWD), which is the time interval between the transmission of the data packet and its reception at the receiver side.

The number of packets transmitted increases until the growing one way delay; when this happens it means that the packet transmission rate is greater than the available bandwidth provided by the radio access network.

Figure 3 shows the above mentioned procedure: when the transmission rate $\mathrm{I}$ is compatible with the available bandwidth (A), i.e. $\mathrm{R}<\mathrm{A}$, one way delay shows an almost constant trend; however, when the packet rate from the source to the destination is greater than the available bandwidth, i.e. $\mathrm{R}>\mathrm{A}$, one way delay increases because of the TCP congestion window mechanism. 

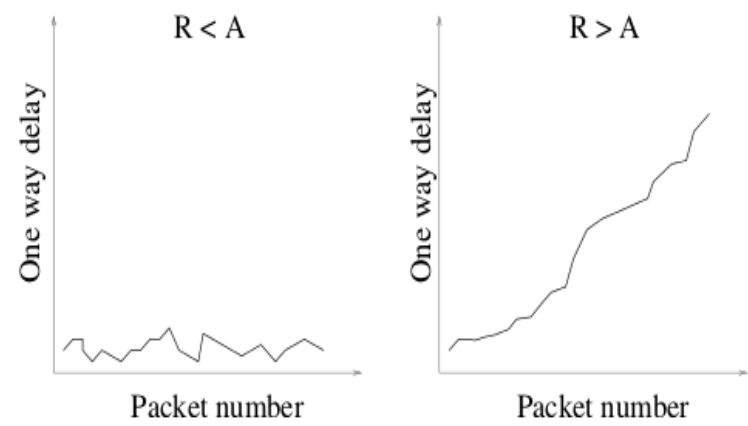

Figure 3. One way delay values of the $\mathrm{i}^{\text {th }}$ packet train in the SloPS technique [3].

In PathLoad the measurement is obtained by iterative sending of a series of K packets of L bit each of which is transmitted during an interval of $T$ seconds. In such a way the transmission rate is equal to $\mathrm{R}=\mathrm{L} / \mathrm{T}$ [bit/s]. Each packet of the series has a timestamp to indicate when the data packet has been created and sent to the receiver node. Once the destination node receives the packets stream it compares the arrival time $\left(\mathrm{A}_{\mathrm{i}}\right)$ and the sending time $\left(\mathrm{S}_{\mathrm{i}}\right)$ of the packets in order to calculate the one way delay of the $i^{\text {th }}$ stream, i.e. $D_{i}=A_{i}-S_{i}$.

The sender and the receiver communicate according to the client-server paradigm in order to establish the available bandwidth. When one way delay at the destination side increases, in fact, the receiver process notifies the sender that behaves as follows:

a. If $\mathrm{R}(\mathrm{i})<\mathrm{A}$, the source process will send the following packets stream at $\mathrm{R}(\mathrm{i}+1)>\mathrm{R}(\mathrm{i})$

b. If $\mathrm{R}(\mathrm{i})>\mathrm{A}$, the source process will send the following packets stream at $\mathrm{R}(\mathrm{i}+1)<\mathrm{R}(\mathrm{i})$

Furthermore, the rate of the stream (i+1) is established as follows [19]:

1. Two start parameters, $R_{\min }$ edR $R_{\max }$, are initialized equal to zero and equal to the ideal maximum throughput provided by the connection Rmax;

$\circ \quad$ If $\mathrm{R}(\mathrm{i})<\mathrm{A}$ then $\mathrm{R}_{\min }=\mathrm{R}(\mathrm{i})$

- If $\mathrm{R}(\mathrm{i})>\mathrm{A}$ then $\mathrm{R}_{\max }=\mathrm{R}(\mathrm{i})$

2. $\mathrm{R}(\mathrm{i}+1)=\left(\mathrm{R}_{\max }-\mathrm{R}_{\min }\right) / 2$

The iterative process ends when $R_{\max }-R_{\min }<w$, where $w$ indicates the value depending on the precision of the bandwidth evaluation procedure (obviously, the greater the $\mathrm{w}$ the less accurate the evaluation but, at the same time, the faster the convergence period required by the algorithm).

PathChirp is based on the same principle but, in this case, the algorithm does not need to send multiple trains of equally spaced data packets increasing at each step the transmission rate. PathChirp is based on the sending of trains of packets, called chirps, characterized by the fact that they are not equally spaced but, vice versa, the interval time between a packet and the next one gradually decreases according to the exponential distribution.

\section{Performance Evaluation}

\section{A.Description of the Testbed}

Tests were carried out in mobility, along predetermined paths and identifying for each path 10 test points where the measurements were taken. 
Tests have been classified into three scenarios which are considered to be of particular interest, especially for the study of how Throughput, Round Trip Time (RTT) and Standard deviation of RTT are related to the RSSI value: the first results refer to the condition of a high quality of the received signal in a high density of mobile users area; the second scenario, instead, refers to an urban context where the areas of particular concentration of users have not been crossed; finally, the last scenario proposes the measurements carried out in a extra-urban environment, where the received signal levels were very poor.

\section{B.Metrics}

The metrics taken into consideration to compare the duplication system and the commonly used single streaming transmissions can be summarized as follows.

To give a first evaluation of the QoS offered by the mobile radio access network, in our analysis, we focused on the measurement of the following parameters:

$\checkmark \quad$ RSSI (Received Signal Strength Indicator)

$\checkmark$ Throughput

$\checkmark$ Throughputevaluationlatency

$\checkmark$ Round Trip Time

$\checkmark \operatorname{Mdev}($ standard deviation of RTT)

\section{C.RSSI}

In telecommunications, received signal strength indicator (RSSI) is a measurement of the power present in the received radiosignal. RSSI is usually invisible to a user of the receiving device. However, because signal strength can vary greatly, it has an impact on the functionality in wireless networking.

A mobile phone signal is the signal strength received by a mobile phone from a cellular network (on the downlink). This parameter can depend on several factors, such as the distance from the Base Station, any obstacle such buildings and trees, etc. So the signal strength can vary greatly.

Typically RSSI is measured in $\mathrm{dBm}$. Another way to express RSSI is the Arbitrary Strength Unit (ASU).Arbitrary Strength Unit (ASU) is an integer value proportional to the received signal strength measured by the mobile phone.It is possible to map the real signal strength measured in $\mathrm{dBm}$ in ASU by means of mathematical relationship depending on the radio access technologyError! Reference source not found..

\section{D.Throughput:}

This parameter represents the average end-to-end bandwidth between source and destination of data packet communication; it is possible to evaluate the average and/or the instantaneous value of the parameter that will have more or less impact according to the type of user application. Throughput is the key performance indicator in case of data transfer and one of the most important indicators in case of multimedia content delivery applications such as real-time audio and video delivery services, i.e. video surveillance, VoIP, private communications system and so on. 


\section{E.Throughput evaluation latency}

In scenarios characterized by high user's mobility or in other cases where the radio access network performance evaluation is carried out by using high mobility vehicles such as drones, the time needed to evaluate the throughput plays a key role in order to implement an effective and efficient measurement campaign; with this scope two well known throughput evaluation methods have been proposed under this perspective, comparing the time needed to converge with the estimated value and the latter with the value obtained by using the traditional file transfer protocol procedure.

\section{F.RTT}

The measurements of the Round Trip Time were carried out using the ICMP protocol. To get a fairly accurate measurement of RTT, each value was obtained by sending 30 packets with an interval of $0.2 \mathrm{sec}$ from each other, for each of them RTT was calculated and finally the average value was estimated.

\section{G.MDEV}

It is the standard deviation, essentially the average measurement of how far each ping RTT is situated from the mean RTT. The higher mdev is, the more variable the RTT is (over time).This parameter, calculated using the same set of ICMP packets sent to the calculation of the average RTT, is an indicator of the homogeneity of the measured delays: the lower the value, the higher the homogeneity level of the delays.

\section{A.Results}

In this section the results obtained by the prototype are illustrated and the conclusions about the behavior of the measurement system are drawn.

The first test campaign is concerned with the assessment of the bandwidth estimation algorithms; as previously mentioned PathLoad and PathChirp were installed on board the prototype and their performances were compared. More specifically, 20 tests were carried out at different points in the urban area of Catania; the first 10 points characterized by a high signal to noise ratio and the second 10 points characterized by low signal-to-noise ratio. For each point, both tools were run for 25 seconds and the detected bandwidth measurements were compared with the results obtained using one proprietary script based on the detection of the effective end-to-end bandwidth by means of the common file transfer protocol. The two algorithms have been compared within the context of traditional wired IP networks, showing that PathChirp offers better performance if compared to PathLoad; the goal of this test was to verify if such behavior was the same also in the context of mobile access network. The results found confirm that PathChirp bandwidth evaluation is more consistent with the value experienced by the end user than the one performed by means of PathLoad. In particular, Table 1 shows the bandwidth values measured by means of the two algorithms and by means of a common file transfer protocol measurement mechanism.PathChirp outperforms PathLoad in all tests: for the twenty performed measurements, each of duration equal to 25 seconds, the average percentage error equaled $21 \%$ for PathChirp and $56 \%$ while running PathLoad. 
International Journal of Wireless \& Mobile Networks (IJWMN) Vol. 8, No. 3, June 2016

Table 1. PathLoad and PathChirp comparison.

\begin{tabular}{|c|c|c|c|c|c|}
\hline Test & $\begin{array}{c}\text { PathChirp } \\
{[\mathrm{Mb} / \mathrm{s}]}\end{array}$ & $\begin{array}{c}\text { PathLoad } \\
{[\mathrm{Mb} / \mathrm{s}]}\end{array}$ & $\begin{array}{c}\text { FTP } \\
{[\mathrm{Mb} / \mathrm{s}]}\end{array}$ & $\begin{array}{c}\text { P.Chirp } \\
\text { vs FTP } \\
{[\%]}\end{array}$ & $\begin{array}{c}\text { P.Load } \\
\text { vs FTP } \\
{[\%]}\end{array}$ \\
\hline 1 & 1.13 & 0.45 & 2.1 & 46,2 & 78,6 \\
\hline 2 & 1.17 & 0.48 & 1.4 & 16,4 & 65,7 \\
\hline 3 & 0.93 & 0.92 & 1.02 & 8,8 & 9,8 \\
\hline 4 & 0.92 & 0.5 & 0.73 & 26,0 & 31,5 \\
\hline 5 & 0.87 & 0.5 & 1.44 & 39,6 & 65,3 \\
\hline 6 & 0.82 & 0.57 & 1.09 & 24,8 & 47,7 \\
\hline 7 & 0.67 & 0.47 & 0.92 & 27,2 & 48,9 \\
\hline 8 & 0.96 & 0.29 & 1.01 & 5,0 & 71,3 \\
\hline 9 & 0.99 & 0.4 & 1.21 & 18,2 & 66,9 \\
\hline 10 & 0.78 & 0.67 & 1.46 & 46,6 & 54,1 \\
\hline 11 & 1.02 & 0.39 & 1.04 & 1,9 & 62,5 \\
\hline 12 & 0.8 & 0.36 & 1.01 & 20,8 & 64,4 \\
\hline 13 & 1.03 & 0.39 & 1.16 & 11,2 & 66,4 \\
\hline 14 & 1.04 & 0.42 & 0.85 & 22,4 & 50,6 \\
\hline 15 & 0.99 & 0.42 & 0.85 & 16,5 & 50,6 \\
\hline 16 & 1.02 & 0.5 & 1.05 & 2,9 & 52,4 \\
\hline 17 & 1.04 & 0.4 & 1.4 & 25,7 & 71,4 \\
\hline 18 & 1.02 & 0.5 & 1.05 & 2,9 & 52,4 \\
\hline 19 & 1.1 & 0.42 & 0.85 & 29,4 & 50,6 \\
\hline 20 & 0.8 & 0.36 & 1.01 & 20,8 & 64,4 \\
\hline
\end{tabular}

Once found that the "Pathchirp" technique best approximates the throughput measured with an FTP session and converges quickly with this value; thus, the following results have been proposed in connection to the three previously mentioned scenarios.

Table 2 shows the results obtained in a scenario with a high level of received signal and high concentration of mobile users. Table 3 shows the results obtained in an urban scenario.

Finally, Table 4 shows the results obtained in an extra-urban scenario where the received signal level was very weak.

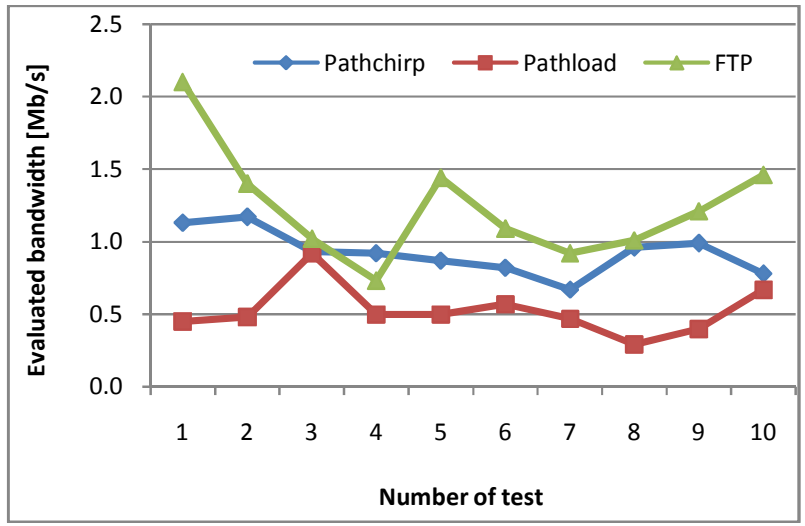

Figure 4 (a)Bandwidth evaluation with PathLoad and PathChirp compared with the effective value measured by means of a FTP session for the first 10 test points 
International Journal of Wireless \& Mobile Networks (IJWMN) Vol. 8, No. 3, June 2016

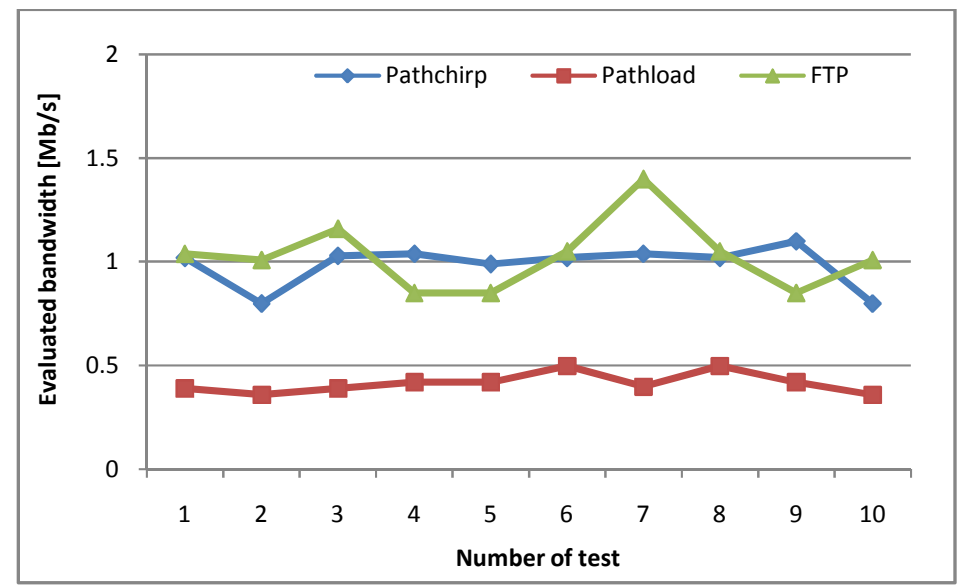

Figure 4 (b) Bandwidth evaluation with PathLoad and PathChirp compared with the effective value measured by means of a FTP session for the last 10 test points

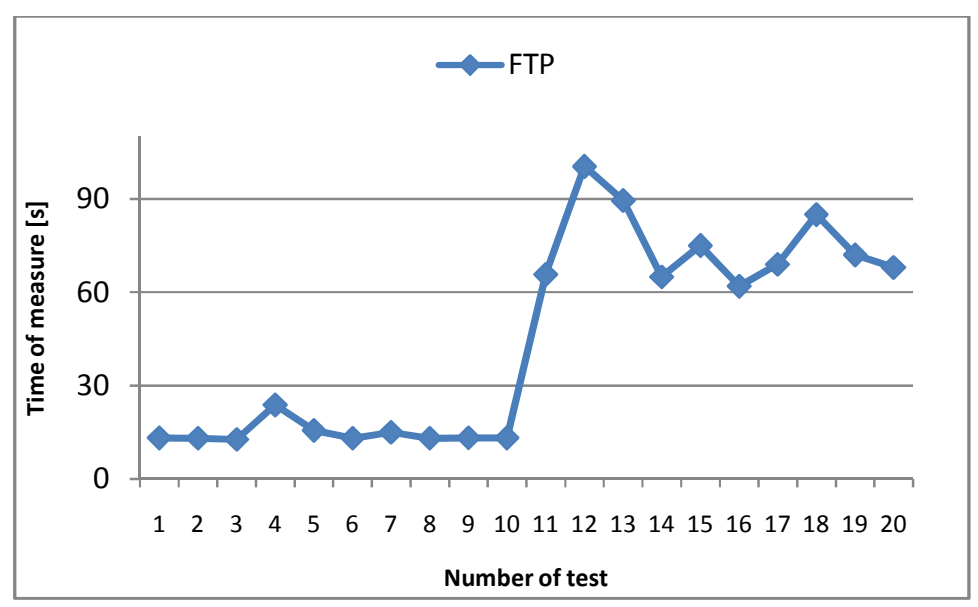

Figure 5 Measurement time for FTP technique 
International Journal of Wireless \& Mobile Networks (IJWMN) Vol. 8, No. 3, June 2016

Table 2. High users density urban area.

\begin{tabular}{|c|c|c|c|c|c|c|}
\hline TEST & \multicolumn{2}{|c|}{ GPS Coordinates } & \multirow{2}{*}{$\begin{array}{c}\begin{array}{c}\text { RSSI } \\
\text { (ASU) }\end{array} \\
22\end{array}$} & \multirow{2}{*}{$\begin{array}{c}\begin{array}{c}\text { Average RTT } \\
\text { (ms) }\end{array} \\
135,526\end{array}$} & \multirow{2}{*}{$\begin{array}{c}\text { MDEV } \\
\text { (ms) } \\
7,6\end{array}$} & \multirow{2}{*}{$\begin{array}{c}\begin{array}{c}\text { Bandwidth } \\
\text { (Mbps) }\end{array} \\
2,172\end{array}$} \\
\hline \multirow[t]{2}{*}{1} & lat: & 37,526513 & & & & \\
\hline & long: & 15,072656 & & & & \\
\hline \multirow[t]{2}{*}{2} & lat: & 37,5267981 & 16 & 133,983 & 13,815 & 2,172 \\
\hline & long: & 15,0750943 & & & & \\
\hline \multirow[t]{2}{*}{3} & lat: & 37,5265764 & 27 & 170,375 & 28,162 & 1,5456 \\
\hline & long: & 15,0785617 & & & & \\
\hline \multirow[t]{2}{*}{4} & lat: & 37,5251086 & 18 & 142,315 & 14,863 & 1,8864 \\
\hline & long: & 15,0747177 & & & & \\
\hline \multirow[t]{2}{*}{5} & lat: & 37,5268042 & 26 & 132,088 & 0,955 & 2,1264 \\
\hline & long: & 15,0696735 & & & & \\
\hline \multirow[t]{2}{*}{6} & lat: & 37,5284868 & 26 & 133,116 & 10,2 & 2,1368 \\
\hline & long: & 15,0689631 & & & & \\
\hline \multirow[t]{2}{*}{7} & lat: & 37,5333923 & 23 & 132,658 & 11,299 & 1,9496 \\
\hline & long: & 15,0677091 & & & & \\
\hline \multirow[t]{2}{*}{8} & lat: & 37,5366734 & 20 & 143,137 & 20,115 & 2,5368 \\
\hline & long: & 15,0674017 & & & & \\
\hline \multirow[t]{2}{*}{9} & lat: & 37,5382284 & 17 & 185,965 & 14,726 & 2,0584 \\
\hline & long: & 15,0706793 & & & & \\
\hline \multirow[t]{2}{*}{10} & lat: & 37,5293026 & 28 & 134,266 & 15,891 & 2,1368 \\
\hline & long: & 15,076781 & & & & \\
\hline
\end{tabular}

Table 3. Urban area.

\begin{tabular}{|c|c|c|c|c|c|c|}
\hline TEST & \multicolumn{2}{|c|}{ GPS Coordinates } & \multirow{2}{*}{$\begin{array}{c}\begin{array}{c}\text { RSSI } \\
\text { (ASU) }\end{array} \\
19\end{array}$} & \multirow{2}{*}{\begin{tabular}{|c}
$\begin{array}{c}\text { Average RTT } \\
\text { (ms) }\end{array}$ \\
175.827
\end{tabular}} & \multirow{2}{*}{$\begin{array}{c}\text { MDEV } \\
\text { (ms) } \\
18.077\end{array}$} & \multirow{2}{*}{\begin{tabular}{|c}
$\begin{array}{c}\text { Bandwidtr } \\
\text { (Mbps) }\end{array}$ \\
2,096
\end{tabular}} \\
\hline 1 & lat.: & 37,527555 & & & & \\
\hline & long.: & 15,098745 & & & & \\
\hline \multirow[t]{2}{*}{2} & lat.: & 37,527585 & 16 & 142 & 17.397 & 2,1024 \\
\hline & long.: & 15,0859046 & & & & \\
\hline \multirow[t]{2}{*}{3} & lat.: & 37,5238828 & 31 & 140.202 & 12.047 & 2,168 \\
\hline & long.: & 15,1158588 & & & & \\
\hline \multirow[t]{2}{*}{4} & lat.: & 34,546725 & 11 & 132.032 & 5.859 & 1,164 \\
\hline & long.: & 15,1405733 & & & & \\
\hline \multirow[t]{2}{*}{5} & lat.: & 37,5487245 & 14 & 175.673 & 52.335 & 1,7672 \\
\hline & long.: & 15,1429347 & & & & \\
\hline \multirow[t]{2}{*}{6} & lat.: & 37,553786 & 24 & 133.783 & 6.978 & 2,1024 \\
\hline & long.: & 15,14591 & & & & \\
\hline \multirow[t]{2}{*}{7} & lat.: & 37,553274 & 11 & 131.601 & 4.636 & 1,8432 \\
\hline & long.: & 15,144885 & & & & \\
\hline \multirow[t]{2}{*}{8} & lat.: & 37,54374 & 17 & 134.326 & 10.867 & 2,1008 \\
\hline & long.: & 15,141795 & & & & \\
\hline \multirow[t]{2}{*}{9} & lat.: & 37,525927 & 20 & 132.553 & 4.743 & 2,096 \\
\hline & long.: & 15,078637 & & & & \\
\hline \multirow[t]{2}{*}{10} & lat.: & 37,526034 & 17 & 143.901 & 19.931 & 2,0984 \\
\hline & Iong.: & 15,078421 & & & & \\
\hline
\end{tabular}


Table 4. Suburban area.

\begin{tabular}{|c|c|c|c|c|c|c|}
\hline TEST & \multicolumn{2}{|c|}{ GPS Coordinates } & \multirow{2}{*}{$\begin{array}{c}\begin{array}{c}\text { RSSI } \\
\text { (ASU) }\end{array} \\
5\end{array}$} & \multirow{2}{*}{$\begin{array}{c}\text { Average RTT } \\
\text { (ms) }\end{array}$} & \multirow{2}{*}{$\begin{array}{c}\text { MDEV } \\
\text { (ms) } \\
14,922\end{array}$} & \multirow{2}{*}{\begin{tabular}{|c|}
$\begin{array}{c}\text { Bandwidth } \\
\text { (Mbps) }\end{array}$ \\
0,4208
\end{tabular}} \\
\hline \multirow[t]{2}{*}{1} & lat & 37,5677718 & & & & \\
\hline & long & 15,0046058 & & & & \\
\hline \multirow[t]{2}{*}{2} & lat & 37,5684959 & 3 & 462,014 & 621,078 & 0 \\
\hline & long & 15,0039841 & & & & \\
\hline \multirow[t]{2}{*}{3} & lat & 37,5692652 & 3 & 367,022 & 521,051 & 0 \\
\hline & long & 15,0031255 & & & & \\
\hline \multirow[t]{2}{*}{4} & lat & 37,5674959 & 1 & Unreachable & Unreachable & 0 \\
\hline & long & 15,0054827 & & host net & host net & \\
\hline \multirow[t]{2}{*}{5} & lat & 37,5677718 & 3 & Unreachable & Unreachable & 0 \\
\hline & long & 15,0046058 & & host net & host net & \\
\hline \multirow[t]{2}{*}{6} & lat & 37,5660917 & 3 & Unreachable & Unreachable & 0 \\
\hline & long & 15,003075 & & host net & host net & \\
\hline \multirow[t]{2}{*}{7} & lat & 37,5644837 & 5 & 141,27 & 26,645 & 0,2752 \\
\hline & long & 15,0015622 & & & & \\
\hline \multirow[t]{2}{*}{8} & lat & 37,5675927 & 5 & 152,362 & 28,733 & 0,2896 \\
\hline & long & 15,0026377 & & & & \\
\hline \multirow[t]{2}{*}{9} & lat & 37,5695428 & 5 & 162,457 & 27,232 & 0,3088 \\
\hline & long & 15,0039383 & & & & \\
\hline \multirow[t]{2}{*}{10} & lat & 37,5645331 & 5 & 159,122 & 32,452 & 0,4256 \\
\hline & long & 15,0037947 & & & & \\
\hline
\end{tabular}

To facilitate the understanding of how the RSSI values influence the other Key Performance Indicators (throughput, RTT and MDEV) we have calculated as these parameters were correlated with the RSSI.

The level of correlation was calculated by means of the Pearson correlation coefficient.

Given two statistical variables, $\mathrm{X}$ and $\mathrm{Y}$, the Pearson correlation index is defined as their covariance divided by the product of the standard deviations of the two variables:

$$
\rho_{x y}=\frac{\sigma_{x y}}{\sigma_{x} \sigma_{y}} \frac{\sigma_{x y}}{\sigma_{x} \sigma_{y}}
$$

where $\sigma_{x y}$ is the covariance between $\mathrm{X}$ and $\mathrm{Y}$, and $\sigma_{x}$ and $\sigma_{y}$ are the two standard deviations. The Pearson correlation coefficient always takes values between -1 and 1

$$
-1 \leq \rho_{x y} \leq 1
$$

Generally, we can distinguish three types of correlation.

- if $\rho_{x y}>0$, variables $\mathrm{X}$ and $\mathrm{Y}$ are called directly correlated;

- if $\rho_{x y}=0$, variables $\mathrm{X}$ and $\mathrm{Y}$ are uncorrelated;

- if $\rho_{x y}<0$, variables $X$ and $Y$ are called inversely correlated.

In addition, for direct correlation (and similarly for inverse correlation) we can distinguish:

- if $0<\rho_{x y}<0.3$ we have a weak correlation;

- if $0.3<\rho_{x y}<0.7$ we have a moderate correlation;

- if $\rho_{x y}>0.7$ we have a strong correlation. 
International Journal of Wireless \& Mobile Networks (IJWMN) Vol. 8, No. 3, June 2016

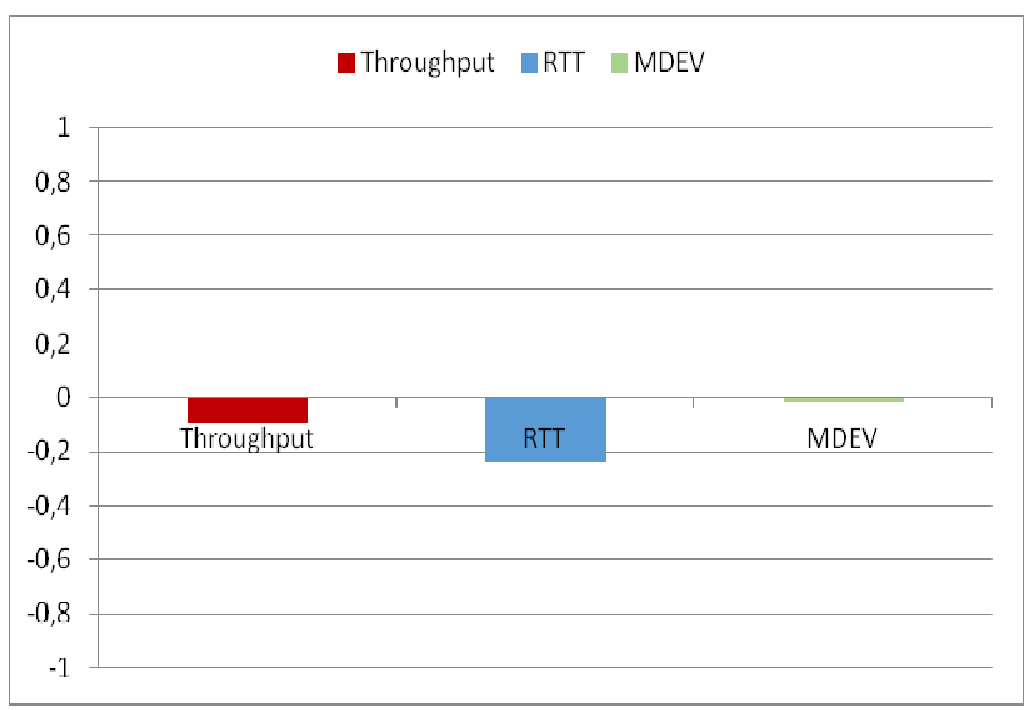

Figure 6: Correlation coefficient between RSSI and Throughput, RTT and MDEV for scenario 1

From the results obtained we can evince how, in the case of the first test (Figure 6), contrary to what was expected, there seems to be no correlation between the values of power and bandwidth.As already mentioned, these measurements were carried out in correspondence of high RSSI values and, especially, in an area with a high concentration of mobile users, in a condition of a high level of commitment of the Mobile Network resources. Consequently, in these cases, we should consider some additional variables not foreseeable in advance that considerably influence the measured parameters.

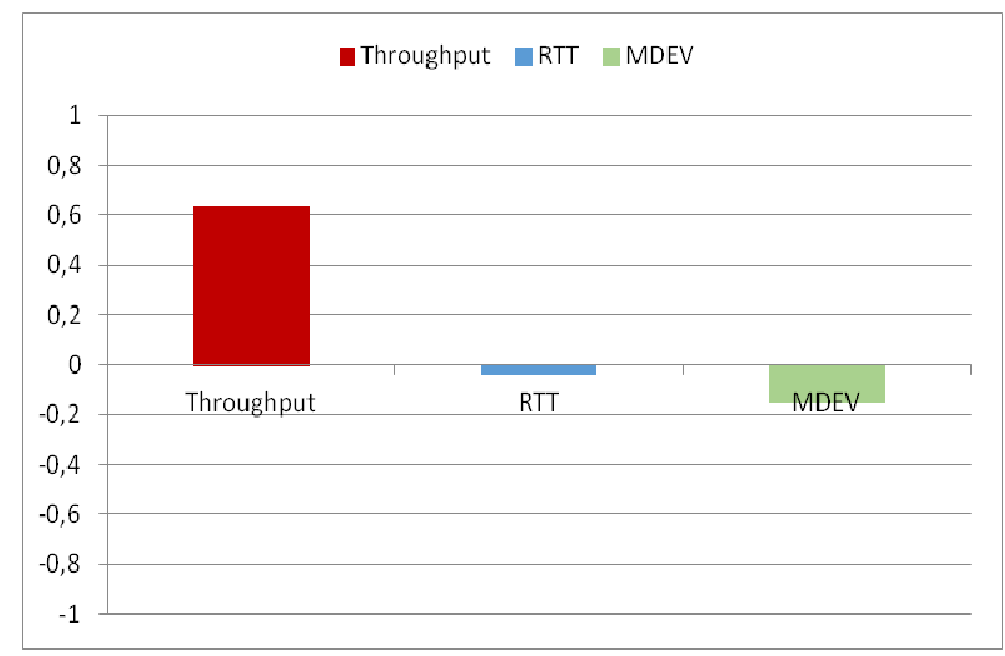

Figure 7: Correlation coefficient between RSSI and Throughput, RTT and MDEV for scenario 2 


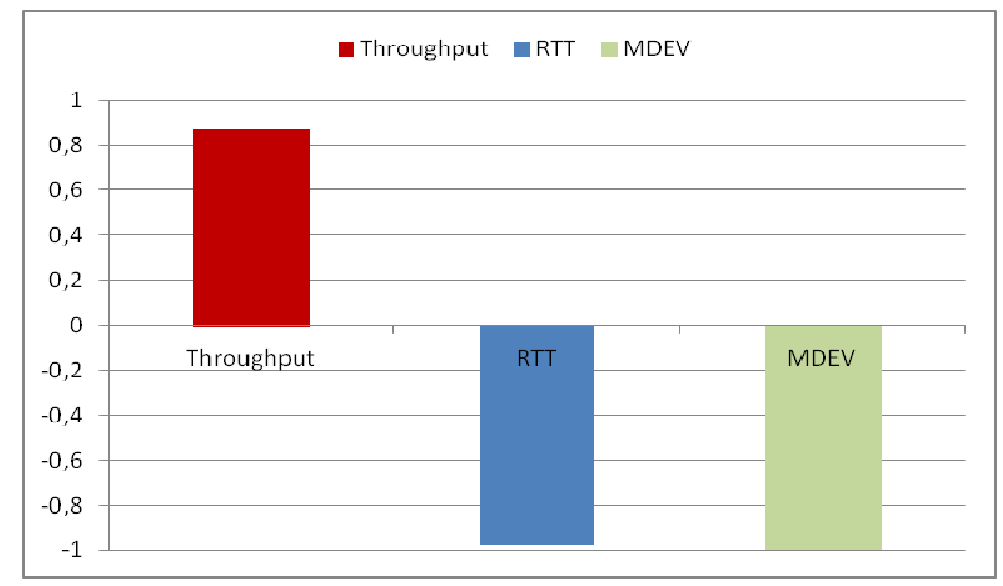

Figure 8: Correlation coefficient between RSSI and Throughput, RTT and MDEV for scenario 3

These results certainly deserve further study, but seem to be enough to indicate that, in this case, it is not the signal strength received to limit the available bandwidth, and neither to influence the Round Trip Time values and their deviation standard.

Instead, by analyzing the results obtained in relation to the second series of measurements (Figure 7), we can see that, in this case, a higher correlation value was reached between RSSI and Available Bandwidth. In this case, the tests were carried out in a normal urban scenario, without crossing particularly "crowded" or "isolated" areas. Along the route, in addition, we could experience a range of variability of the much larger RSSI. As we can see, when the RSSI values decline to relatively low values (test points 4,5 , and 7), the measured bandwidth value drops considerably. It is evident that when the RSSI drops below certain values it especially affects the value of available bandwidth.

As regards the values of RTT and standard deviation, they are still clearly uncorrelated.The latest results proposed are related to a suburban area with very poor cell coverage (Figure 8). In fact, as one can evince from the RSSI values, the received signal levels are very scarce.

Due to the poor conditions of coverage, measurements often failed, and at times the bandwidth measurement took too long. By convention, it was decided to put the value of available bandwidth to zero when the measurement time exceeded $300 \mathrm{sec}$. It is also quite clear that very low RSSI values ( $<=3 \mathrm{ASU}$ ) lead to a significant instability of Internet connectivity.

In this scenario, by observing the correlation values we can see that there is a strong correlation between RSSI and Available Bandwidth, RTT and MDEV (direct correlation between RSSI and available bandwidth, inverse correlation between RSSI and RTT and between RSSI and MDEV).

From the results obtained from the three series of proposed measurements we can conclude that there is a correlation between the RSSI and the measured parameters which becomes stronger when the RSSI values drop to gradually lower values.

In good received signal level conditions, the variables are decidedly uncorrelated since, in this case, it was determined that it is certainly not the signal power to influence the network parameters measured but other parameters (e.g. traffic conditions, momentary network congestion, number of mobile users in the cell, etc.). 


\section{Conclusions And Future Works}

The present paper provides an extended performance evaluation method of Quality of Service in mobile Internet access. In particular, the paper proposes an algorithm for the throughput estimate based on PathChirp technique, which we have seen to be preferable to PathLoad technique as it is able to better approximate measurements using the FTP technique and converges with the desired values in a significantly more rapid way. The convergence speed is a decisive factor especially if applied to a context in which the measurements are taken by the use of drones. The use of drones definitely has several advantages:

- possibility of moving in 3 dimensions and consequently greater spatial resolution of the measurements;

- ability to record measurements in not easily accessible areas;

- carrying out measurements near the radio base station.

In contrast, a problem arises from the autonomy of the drone. Measurements must therefore be carried out respecting certain timing constraints precisely due to the autonomy of the drone in flight.During a single flight session it is possible to obtain several measurement points and therefore have a more precise estimate of the QoS and increased spatial resolution.

\section{ACKNOWLEDGMENT}

We would like to thank HELI-LAB S.r.l., drone aerial filming company, and VICOSYSTEMS S.r.l., company of telecommunications equipment design that has provided the modem-router multi-sensor on board the drone.

\section{REFERENCES}

[1] Vinay J. Ribeiro, Rudolf H. Riedi, Richard G. Baraniuk Jiri Navratil, Les Cottrell, "pathChirp: Efficient Available Bandwidth Estimation for Network Paths", in Passive and Active Measurement Workshop, April2003

[2] Manish Jain, ConstatninosDovrolis, "Pathload: a measurement tool for end-to-end availabla bandwidth", in Proc. Of Passive and Active Measurements (PAM) Workshop, 2002.

[3] R.S. Prasad, M.Murray, C.Dovrolis, K.Claffy, "Bandwidth Estimation: metrics, measurement techniques and tools", IEEE Network, Nov 2003

[4] C. Eschmann, C.-M. Kuo, C.-H. Kuo, C. Boller," High-resolution multisensory infrastructure inspection with unmanned aircraft systems", International Archives of the Photogrammetry, Remote Sensing and Spatial Information Sciences, Volume XL-1/W2, 2013 UAV-g2013, 4 - 6 September 2013, Rostock, Germany

[5] Md. Arafatur Rahman, "Enabling drone communications with WiMAX Technology", Information, Intelligence, Systems and Applications, IISA 2014, The 5th International Conference on

[6] LudovicApvrille, Yves Roudier, Tullio Joseph Tanzi, “Autonomous drones for disasters management: Safety and security verifications",Radio Science Conference (URSI AT-RASC), 2015 1st URSI Atlantic

[7] Daniel Câmara, "Cavalry to the rescue: Drones fleet to help rescuers operations over disasters scenarios", Antenna Measurements \& Applications (CAMA), 2014 IEEE Conference on

[8] LudovicApvrille, TullioTanzi ; Jean-Luc Dugelay, "Autonomous drones for assisting rescue services within the context of natural disasters", General Assembly and Scientific Symposium (URSI GASS), 2014 XXXIth URSI

[9] R.Viguier,C.,C.Lin ; K.Swaminathan, A.Vega, A.Buyuktosunoglu; S.Pankanti, P.Bose, H.Akbarpour, F.Bunyak ; K.Palaniappan, G. Seetharaman, "Resilient mobile cognition:Algorithms, innovations, and architectures - - Computer Design (ICCD), 2015 33rd IEEE International Conference on 
[10] AislanGomideFoina Raja Sengupta ; Patrick Lerchi ; Zhilong Liu ; Clemens Krainer, "Drones in smart cities: Overcoming barriers through air traffic control research", 2015 Workshop on Research, Education and Development of Unmanned Aerial Systems (RED-UAS)

[11] UtkarshGoel, Mike P. Wittie, Kimberly C. Claffy, and Andrew Le , "Survey of End-to-End Mobile Network Measurement Testbeds, Tools, and Services”, IEEE Communications Surveys \& Tutorials (Volume:18, Issue: 1 ), Ott. 2015

[12] AshkanNikravesh, David R. Choffnes, Ethan Katz-Bassett, Z. Morley Mao, and Matt Welsh, "Mobile Network Performance from User Devices: A Longitudinal, Multidimensional Analysis", 15th International Conference, PAM 2014, Los Angeles, CA, USA, March 10-11, 2014, Proceedings

[13] http://company.nokia.com/en/news/press-releases/2015/07/07/nokia-networks-du-first-in-uae-to-usetelco-drones-for-better-network-planning-faster-optimization

[14] http://www.pcengines.ch/alix.htm

[15] F. Beritelli, A. La Corte, C. Rametta and F. Scaglione "A Cellular Bonding and Adaptive Load Balancing based Multi-SIM Gateway for Mobile ad Hoc and Sensor Network" International Journal on Ad Hoc Networking Systems (IJANS), Vol. 5, No. 3, July 2015

[16] F. Beritelli, A. La Corte, G. Lo Sciuto, C. Rametta, F. Scaglione, "Adaptive VPN BondingTechnique for Enhancing Dual-SIM Mobile Internet Access". Proc. of the International Symposium for Young Scientists in Technology, Engineering and Mathematics (SYSTEM) 2015, Catania, Italy, September 27-29, 2015.

[17] http://www.zeroshell.net

[18] http://www.python.org/

[19] Manish Jain, ConstantinosDovrolis, "End-to-end Available Bandwidth: Measurements, Methodology, Dynamic and Relation with TCP Throughput", IEEE/ACM Transactions on Networking, Ago 2003.

[20] 3GPP TS 27.007 version 8.5.0 Release 8

\section{AuTHORS}

Francesco Beritelli received the Laurea degree in electronic engineering and the $\mathrm{Ph} . \mathrm{D}$. degree in electronics, computer science, and telecommunications engineering from the University of Catania, Catania, Italy, in 1993 and in 1997, respectively. From 1997 to 2000 in collaboration with CSELT (now Telecom Italia LAB) took an active part in international ITU-T standardization meetings. From 1998 he is founding member of the "Multimedia Technologies Institute - MTI", applied research laboratory operating in DSP (Digital Signal Processor) and CTI (Computer Telephony Integration) applications. Currently, from 2002, he is Assistant Professor in the Department of Electric, Electronic and Computer Science Engineering at the

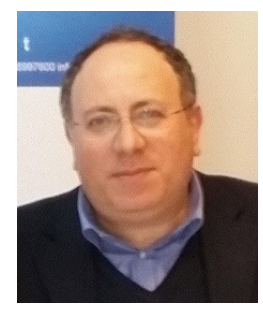
University of Catania. His main research activities are in the area of robust audio and speech signal classification and recognition, variable bit-rate speech coding, and adaptive-rate voice and dual stream transmission for mobile IP telephony applications, QoS in mobile Internet access and drone communications. His interests also include the field of biometric identification and cardiac signal processing. He is member of the Institute of Electrical and Electronic Engineers (IEEE) and the "IEEE Signal Processing Society" since 1996 and has 120 scientific publications, mainly in international journals, books and conference proceedings.

CorradoRametta received the Laurea degree in electronic engineering and the $\mathrm{Ph} . \mathrm{D}$. degree in computer science and telecommunications engineering from the University of Catania, Italy, in 2008 and in 2012, respectively. From 2009 to 2012, he was a Research Engineer in the field of wireless communications with the CNIT (ConsorzioNazionaleInteruniversitario per le Telecomunicazioni). From 2012 he works as post-doc researcher at the University of Catania. His research interests include wireless mesh networks, ad-hoc and sensor networks, modeling and simulation of communications protocols, software defined networking, network functions virtualization and embedded systems for multimedia applications.

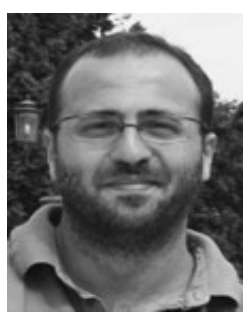


Antonio Raspanti founder of Heli-Lab,drone aerial filming company. His research interests include drone communications and performance evaluation techniques for mobile Internet access.

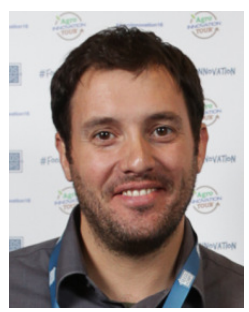

Michele Russo received the Laurea degree in Computer Science Engineering from the University of Catania, Italy, in 2010. His research interests include IoT systems, VoIP Gateway (SIP compliant) and ICT technologies for environmental control and monitoring.

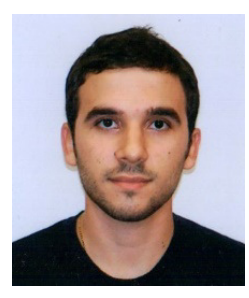

Francesco Scaglionereceived the Laurea degree in Telecommunications Engineeringfrom the University of Catania in 2011. His research interests include embedded systems, VoIP communications and ICT technologies for environmental control and monitoring.

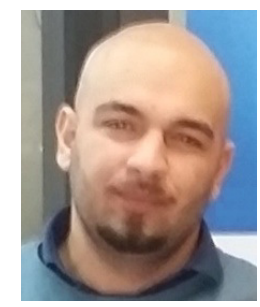

GiuseppeSpallina founder of Heli-Lab,drone aerial filming company. His research interests include drone communications and performance evaluation techniques for mobile Internet access.

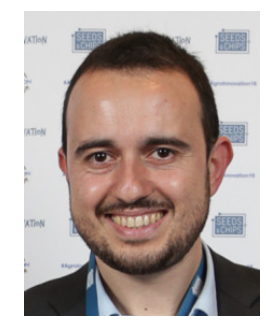

